\title{
Fixed Priority Scheduling of Periodic Tasks with Varying Execution Priority
}

\author{
Michael Gonzalez Harbour \\ Departmento de Electronica \\ Universidad de Cantabria \\ 39005 - Santander, Spain
}

\author{
Mark H. Klein \\ Software Engineering Institute \\ Carnegie Mellon University \\ Pittsburgh, PA 15213
}

\author{
John P. Lehoczky \\ Department of Statistics \\ Carnegie Mellon University \\ Pittsburgh, PA 15213
}

\begin{abstract}
$^{1}$
This paper considers the problem of fixed priority scheduling of periodic tasks where each task's execution priority may vary. Periodic tasks are decomposed into serially executed subtasks, where each subtask is characterized by an execution time and a fixed priority, and is permitted to have a deadline. A method for determining the schedulability of each task is presented along with its theoretical underpinnings. This method can be used to analyze the schedulability of complex task sets which involve interrupts, certain synchronization protocols, nonpreemptible sections and, in general, any mechanism that contributes to a complex priority structure. The method is illustrated with a realistic example.
\end{abstract}

\section{Introduction}

A theoretical treatment of fixed priority scheduling first appeared in 1973 when Liu and Layland [7] introduced the notion of rate monotonic priorities for periodic tasks. They proved the optimality of a rate monotonic priority assignment (for fixed priority scheduling) for the case where task deadlines are coincident with the end of a task's period. They also derived a sufficient condition for the schedulability of task sets

\footnotetext{
${ }^{1}$ Michael Gonzalez is currently on sabbatical as a resident affiliate at the Software Engineering Institute. His research is sponsored in part by the Direccion General de Investigacion Cientifica y Tecnica of the Spanish Government.
}

Mark Klein's research at the Software Engineering Institute is sponsored by the U.S. Department of Defense.

John Lehoczky's research is sponsored in part by the Office of Naval Research under contract number N0014-84-K-0734, in part by the Naval Ocean Systems Center under contract N66001-87-C-0155, and in part by the Federal Systems Division of IBM Corporation under University Agreement Y-278067. that use a rate monotonic priority assignment. Leung and Whitehead [6] later showed the optimality of a deadline monotonic priority assignment for the case where periodic tasks have deadlines that are at or before the end of their periods.

Fixed priority scheduling theory has received renewed consideration over the last five years. This collection of results is proving to be very useful and is gaining popularity as a basis for reasoning about the timing behavior of real-time systems. These results are summarized by Sha, Klein, and Goodenough [14], and Sha and Goodenough [11]. Aperiodic task scheduling has been treated in [15], synchronization requirements treated in $[8,9,13]$, and mode change requirements treated in [10]. In addition, hardware scheduling support has been treated in $[4,12]$, implications for Ada scheduling rules discussed in [2], and schedulability analysis of input/output paradigms discussed in [3].

Naturally, one of the assumptions of fixed priority scheduling is that a task executes at a constant priority during the course of a single period. Yet there are many occasions where this premise is violated. Even in cases where one strives to use a fixed priority assignment for periodic tasks, the task dispatching mechanism may violate this premise since periodic tasks are generally initiated by clock interrupts. Borger, Klein, and Veltre [1] present a schedulability analysis that considers the effects of task dispatching. The basic inheritance protocols also change execution priority as necessary. Sha and Goodenough [11] discuss a mechanism for emulating the priority ceiling protocol by setting the priority of a server task to be one level higher than the priority of all of the server's clients. This represents another instance of varying priorities. It is not uncommon for operating systems to ensure internal consistency by disabling interrupts for short periods of time, effectively creating small intervals of nonpreemptibility, which need to be considered when analyzing the timing behavior of a 
system. Finally, there are many systems that have been developed where a single logical thread of execution comprises a sequence of processing steps that are implemented as a set of tasks that are serially executed at varying priority levels. In these cases task priority may be based upon a deadline or based upon semantic importance. For all of these reasons a formal framework for reasoning about the timing behavior in the context of varying execution priority is needed. This paper presents such a formal framework.

This paper is organized as follows. The remainder of this section presents the framework for the scheduling problem and discusses a difficulty that arises when task priorities vary. Section 2 presents an algorithm and schedulability equations for checking task set schedulability. Section 3 introduces a simple but realistic real-time robotics application and illustrates how one uses the schedulability equations presented in Section 2 . Section 4 proves the method's correctness. Section 5 offers our conclusions.

\subsection{The Framework}

We assume there are $\mathrm{n}$ periodic tasks denoted by $\tau_{1}, \ldots, \tau_{\mathrm{n}}$. Each periodic task $\tau_{\mathrm{i}}$ has a total computation requirement $\left(C_{i}\right)$, a period $\left(T_{i}\right)$, and a deadline $\left(D_{i}\right)$. The computation requirement must be completed by the deadline or a timing fault occurs. In this paper, we assume that each periodic task may be composed of distinct subtasks, each of which may have its own timing requirement. Thus, $\tau_{\mathrm{i}}$ consists of subtasks $\tau_{\mathrm{i} 1}, \ldots, \tau_{\mathrm{im}(\mathrm{i})}$. Each of these subtasks is characterized by a set of parameters. In particular, $\tau_{\mathrm{ij}}$ is characterized by $\left(\mathrm{C}_{\mathrm{ij}}, \mathrm{D}_{\mathrm{ij}}, \mathrm{P}_{\mathrm{ij}}\right)$ where:

- $\mathrm{C}_{\mathrm{ij}}=$ worst-case computation requirement of $\tau_{\mathrm{ij}}$,

- $D_{\mathrm{ij}}=$ deadline of $\tau_{\mathrm{ij}}$ relative to the arrival time of task $\tau_{\mathrm{i}}$, taking 0 to be this arrival time,

- $\mathrm{P}_{\mathrm{ij}}=$ fixed priority level of $\tau_{\mathrm{ij}}$.

We assume that $0 \leq D_{i 1} \leq \ldots \leq D_{i m(i)}=D_{i}$, and we allow either $D_{i m(i)} \leq T_{i}$, or $D_{i m(i)}>T_{i}$. The sum of the execution times associated with each subtask of task $\tau_{i}$ equals $\mathrm{C}_{\mathrm{i}}$. Each activation of a periodic task generates an instance of task execution called a job of that task.

Using this framework, we wish to derive a set of equations by which we can determine if all of the timing requirements of all of these tasks will be met under all possible phasings. We make the following assumptions concerning the execution behavior of any single task:

Assumption 1: Subtasks executing at a given priority level can be preempted by any subtask of higher priority.

Assumption 2: Tasks do not suspend themselves at any instant between their activation and their completion.

Assumption 3: The $(\mathrm{k}+1)^{\mathrm{st}}$ job of $\tau_{\mathrm{i}}$ will not execute until the $\mathbf{k}^{\text {th }}$ job of $\tau_{\mathrm{i}}$ has been completed. Furthermore, any subtask $\tau_{\mathrm{ij}}$ is not ready for execution until subtasks $\tau_{\mathrm{ir}}, 1 \leq \mathrm{r}<\mathrm{j}$ have been completed.

Assumption 4: The time required to perform task scheduling, context swapping, and other overhead is ignored.

There are situations, especially involving interrupts, in which one would want to modify Assumption 3 to allow early subtasks of a later job to interrupt unfinished later subtasks of an earlier job. The analysis given in Section 4 can be modified to handle this case, but we develop the theory specifically for Assumption 3.

To determine the schedulability of a task $\tau_{\mathrm{i}}$, we will apply a transformation to its priority structure to derive its canonical form.

Definition 1: A task is said to be in canonical form if it consists of consecutive subtasks that do not decrease in priority. The canonical form of a task $\tau_{i}$ is another task, $\tau_{\mathrm{i}}^{\prime}$, that is obtained by applying the following algorithm where $P_{\mathrm{ij}}^{\prime}$ denotes the priority of subtask $\tau_{\mathrm{ij}}^{\prime}$.

$$
\begin{aligned}
& P_{i m(i)}^{\prime}=P_{i m(i)} \\
& \text { for } l=m(i) \text { downto } 2 \\
& \quad \text { if } P_{i l}^{\prime}<P_{i l-1} \text { then } P_{i l-1}^{\prime}=P_{i l}^{\prime} \\
& \text { else } P_{i l-1}^{\prime}=P_{i l-1} \\
& \text { end; }
\end{aligned}
$$

After applying the algorithm, consecutive subtasks of the transformed task with equal priority can be combined into a single subtask, if their deadlines are the same.

We will prove in Section 4 that the completion time of a task $\tau_{\mathrm{i}}$ and the completion time of its canonical form $\tau_{i}^{\prime}$ are the same. The canonical form has the advantage of being simpler for the purpose of understanding the worstcase phasing and performing the schedulability analysis.

\subsection{A Difficulty}

The possibility that a task's deadline, $\mathrm{D}_{\mathrm{im}(\mathrm{i})}$, exceeds its period, $T_{i}$, requires one to check more than just the deadline of the first job of a particular task. As shown in [5], all deadlines in a particular time interval called a busy period must be checked. Suppose, however, that $\mathrm{D}_{\mathrm{im}(\mathrm{i})} \leq \mathrm{T}_{\mathrm{i}}, 1 \leq \mathrm{i} \leq \mathrm{n}$, so that a job of task $\tau_{\mathrm{i}}$ must be completed before the next job of $\tau_{\mathrm{i}}$ is ready (assuming task deadlines are met). Consequently, earlier jobs of $\tau_{i}$ do not compete with later jobs, and it would seem that one could follow a standard Liu and Layland approach, namely constructing a worst-case phasing of all other tasks and checking that the first job of $\tau_{\mathrm{i}}$ meets its deadline. The difficulty with this reasoning is that it overlooks another way in which earlier jobs of $\tau_{i}$ can influence later jobs. If an early subtask of $\tau_{\mathrm{i}}$, say $\tau_{\mathrm{ip}}$, has a relatively low priority, $\mathrm{P}_{\mathrm{ip}}$, while a later subtask, $\tau_{\mathrm{ir}}, \mathrm{p}<\mathrm{r}$, has a relatively high priority, $\mathrm{P}_{\mathrm{ip}}<\mathrm{P}_{\mathrm{ir}}$, then $\tau_{\mathrm{ir}}$ can delay a medium priority subtask $\tau_{\mathrm{kq}}, \mathrm{k} \neq \mathrm{i}$ where $\mathrm{P}_{\mathrm{ip}}<\mathrm{P}_{\mathrm{kq}}<\mathrm{P}_{\mathrm{ir}}$. This delay in $\tau_{\mathrm{kq}}$ creates an intermediate phasing which cannot be created as an initial phasing and which can lead to 
longer response times for the next job of subtask $\tau_{i p}$. Consider the following example.

Example: Let $n=2$, where task $\tau_{1}$ has one subtask given by $C_{11}=4, D_{11}=10$, and $T_{1}=10$. Task $\tau_{2}$ has period $\mathrm{T}_{2}=14$ and two subtasks characterized by $\mathrm{C}_{21}=6$, $\mathrm{D}_{21}=14$, and $\mathrm{C}_{22}=2, \mathrm{D}_{22}=14$. Suppose further that $\mathrm{P}_{21}<\mathrm{P}_{11}<\mathrm{P}_{22}$.

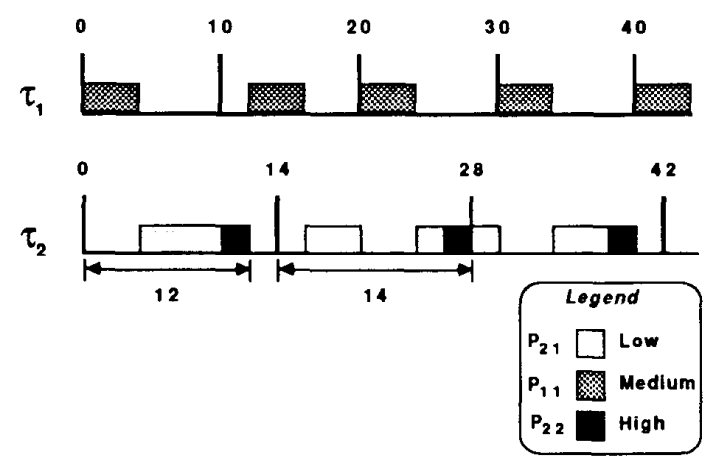

Figure 1: Considering the First Job is Not Sufficient

Under the traditional (Liu and Layland) worst-case phasing shown in Figure 1, the longest response time for $\tau_{22}$ is given by 14 for the second job of $\tau_{2}$, whereas the first job has a response time of 12 . Thus, the longest response time of this task is associated with its second job rather than the first. The cause of this phenomenon is the high priority accorded to $\tau_{22}$ which delays the start of the second job of $\tau_{1}$. This, in turn, creates the long response times for the subtasks of $\tau_{2}$. Note that had $\tau_{i}$ not been divided into two parts, but had been given its rate monotonic priority, then the task set would not be schedulable. We will return to this point in Section 4.5.

\subsection{Busy Period}

The above example shows that one may need to check the deadlines of more than one job of a particular task. The criterion for which deadlines need to be checked is based on the concept of a busy period. The concept of a busy period is well known in queuing theory and was first introduced in real-time scheduling by Lehoczky [5]; however, we need to modify this concept slightly to accommodate the fact that a single task may have subtasks with different priorities. Let $P_{i}=\min \left(P_{i j}, 1 \leq j \leq m(i)\right)$ denote the minimum priority level of all of the subtasks of task $\tau_{\mathrm{i}}$.

Definition 2: A $\tau_{-i}$-idle instant is any time $t$ such that all work of priority $P_{i}$ or higher started before $t$ and all $\tau_{\mathrm{i}}$ jobs also started before $t$ have completed at or before $t$.
Definition 3: A $\tau_{i}$-busy period is an interval of time $[A, B]$ such that both $A$ and $B$ are $\tau_{\mathrm{i}}$-idle instants and there is no time $t \in(A, B)$ such that $\mathrm{t}$ is a $\tau_{\mathrm{i}}$-idle instant.

Intuitively, a $\tau_{\mathrm{i}}$-busy period is a time interval during which the processor is continuously processing at priority level $\mathrm{P}_{i}$ or higher. It is also "self-contained" in the sense that any job of task $\tau_{i}$ that is started during the busy period is also completed during the busy period. Moreover, all work of priority level $P_{i}$ or higher which is ready at some time during the busy period is also finished by the end of the busy period.

\section{A Method for Determining Schedulability}

This section describes a procedure for determining the schedulability of a task set of the general form described in the previous section. We will assume that we are analyzing a single task in the task set, and the method used can be subsequently applied to all of the other tasks.

\subsection{Final Deadline}

First, we will focus on the task's final deadline. Our goal is to determine if task $\tau_{i}$ will meet its final deadline. The general strategy is very similar to that used by Liu and Layland [7] and Lehoczky [5]. One must first find the phasing of the other tasks relative to task $\tau_{i}$ that results in a critical instant for task $\tau_{i}$. A critical instant is a point in time such that if task $\tau_{i}$ is activated at that point, its completion time will be the longest. In order to determine the critical instant phasing, we will first divide the other n-1 tasks into several groups.

2.1.1 Task Groups: Tasks are placed into groups based upon the priorities of their subtasks. A key criterion is the priority of the first subtask relative to the minimum priority of all subtasks of task $\tau_{j}$. For example, a task that starts with a high-priority subtask will eventually be able to preempt the subtask of task $\tau_{i}$ with the minimum priority. On the other hand, a task $\tau_{i}$ that starts with a lower priority subtask will never have this opportunity. Since the task groupings are relative to the priority structure of task $\tau_{i}$, the groups will vary as a function of the task being analyzed.

Recall that $P_{i}=\min \left(P_{i j}, 1 \leq j \leq m(i)\right)$ denotes the minimum priority of all of the subtasks of task $\tau_{i}$. We refer to a sequence of consecutive subtasks as a segment. An $H$ segment comprises a sequence of consecutive subtasks, each of which has a priority equal to or greater than $\mathrm{P}_{\mathrm{i}}$. Note that this is a possibly pessimistic treatment of equal priority subtasks, allowing for a worst-case analysis and thus covers all scheduling policies for handling equal priorities. Similarly, an $L$ segment refers to any set of consecutive subtasks, each of which has priority strictly less than $P_{i}$. In the following description of task types, a " + " denotes one or more patterns. A " 0 " denotes zero or one patterns. An effect due to preemption by a high prior- 
ity first segment will be referred to as a preemption effect. An effect due to a high-priority segment that occurs after a low-priority segment will be referred to as a blocking effect. Figure 1 shows preemption effects of $\tau_{1}$ over $\tau_{2}$ at times $t=0, t=12, t=20$, and $t=30$; a blocking effect of $\tau_{2}$ on $\tau_{1}$ is shown at time $t=10$.

The five types of tasks are:

- Type $1(\mathrm{H})$ tasks may be able to preempt task $\tau_{\mathrm{i}}$ more than once per $\tau_{\mathrm{i}}$-busy period. Each task in this group is used to determine the worst-case completion time for task $\tau_{\mathrm{i}}$.

- Type $2\left((\mathbf{H L})^{+}\right)$tasks are such that each high-priority segment is followed by a low-priority segment. Consequently, each task in this set can preempt task $\tau_{\mathrm{i}}$ only once per $\tau_{\mathrm{i}}$-busy period because the low-priority segments will have to wait until the busy period is complete before they can execute. Depending on types 3 and 4 , one task in this set may be used for its blocking effect rather than its preemption effect.

- Type $3\left((\mathbf{H L})^{+} \mathbf{H}\right)$ differ from the previous type in that they end with a high-priority segment. In general these tasks are treated like tasks of type 2. However, under special circumstances (described below) one task may exhibit both a preemption effect and a blocking effect.

- Type $4\left((\mathbf{L H})^{+} L^{0}\right)$ tasks are solely blocking tasks. Moreover, at most one task in this group can contribute a blocking effect.

- Type 5 (L) tasks have no effect on the completion time of task $\tau_{i}$ and thus can be ignored.

2.1.2 Schedulability Equations: In this section a stepby-step procedure is described for determining if tasks can meet their final deadlines. The procedure entails methodically identifying blocking effects and preemption effects, and constructing equations that account for how these effects contribute to the completion time of task $\tau_{\mathrm{i}}$.

We will assume throughout this section that task $\tau_{i}$ has been transformed to canonical form and that adjacent subtasks of equal priority have been compressed into a single subtask. This results in a task where each subtask has a priority strictly higher than its predecessor. The rest of the tasks remain unchanged.

The procedure determines the completion time of the first subtask of the transformed canonical form. It then iteratively determines the completion of the $(j+1)^{\text {st }}$ subtask as a function of the $j^{\text {th }}$ subtask until the completion time of the final subtask has been determined. This is performed for every job in the $\tau_{\mathrm{i}}$-busy period.

Some notation is needed prior to discussing the procedure. Let $M P_{i j}$ denote the set of tasks that can have more than one preemptive effect relative to the priority of the subtask $\tau_{\mathrm{ij}}$. Recall that $\mathrm{P}_{\mathrm{p}}=\min \left(\mathrm{P}_{\mathrm{pj}}, 1 \leq \mathrm{j} \leq \mathrm{m}(\mathrm{p})\right)$.

$$
M P_{i j}=\left\{\left.\tau_{p}\right|_{p \neq i \wedge P_{p} \geq P_{i j}}\right\}
$$

For example, since $\tau_{\mathrm{i}}$ is assumed to be in canonical form, $\mathrm{MP}_{\mathrm{i} 1}$ is simply the set of type 1 tasks for $\tau_{\mathrm{i}}$. Since the priority of the second subtask of the canonical form is greater than the first, not all of the tasks that are multiply preemptive (i.e., can preempt more than once during a task $\tau_{\mathrm{i}}$-busy period) relative to $\tau_{\mathrm{i} 1}$ will continue to be multiply preemptive during the execution of the second subtask. Consider the example in Figure 1. Task 1 is multiply preemptive until task 2 enters its second subtask; then task 1 can no longer preempt.

Let $S P_{i j}$ be the set of singly preemptive tasks relative to $\tau_{\mathrm{ij}}$. Furthermore, given a task $\tau_{\mathrm{p}} \in \mathrm{SP}_{\mathrm{ij}}$, let $h(p, i, j)$ denote the number of subtasks that comprise the initial $H$ segment of task $\tau_{\mathrm{p}}, \mathrm{p} \neq \mathrm{i}$, relative to subtask $\tau_{\mathrm{ij}}$, where

$$
\begin{aligned}
h(p, i, j)= & \left(h \mid p \neq i \wedge\left(P_{p 1}, \ldots, P_{p h} \geq P_{i j}\right)\right. \\
& \left.\wedge\left(P_{p h+1}<P_{i j}\right)\right)
\end{aligned}
$$

The execution time associated with the leading $\mathrm{H}$ segment of one of these tasks is denoted as:

$$
C_{p}^{h(p, i, j)}=\sum_{k=1}^{h(p, i j)} C_{p k}
$$

In the context of a task $\tau_{\mathrm{p}}$ and a subtask $\tau_{\mathrm{ij}}, h$ will serve as notational shorthand for $h(p, i, j)$ and $C_{p}^{h}$ for $\mathrm{C}_{\mathrm{p}}^{\mathrm{h}(\mathrm{p}, \mathrm{i}, \mathrm{j})}$.

The stepwise procedure for determining if task $\tau_{i}$ can meet its final deadline follows.

Step 1: Find the worst-case phasing for the other n-1 tasks.

In this step tasks other than $\tau_{\mathrm{i}}$ are placed into the groups defined in the previous section, $\mathrm{MP}_{\mathrm{i} 1}$ and $\mathrm{SP}_{\mathrm{i} 1}$ are determined, and then the blocking term, $B_{i}$, is identified.

1. Let $\mathrm{MP}_{\mathrm{i} 1}$ be the set of type 1 tasks for $\tau_{\mathrm{i}}$ and set $S P_{i 1}$ to the union of type 2 and type 3 tasks.

2. Determine the longest $\mathrm{H}$ segment of the type 4 tasks. Denote the length of this as B'.

3. For each type 2 and 3 task, denote the length of the longest inner blocking $\mathrm{H}$ segment (if any) as $\mathrm{U}$, the final blocking $\mathrm{H}$ segment (if any) as $\mathrm{V}$, and the initial $\mathrm{H}$ segment as $\mathrm{W}$. If there is no inner blocking segment then set $U=0$. If there is no final blocking segment then set $\mathrm{V}=0$.

Calculate max(U-W-B', V-B') for each task and choose the task with the largest value (let $\tau_{\mathrm{m}}$ be this task). If this value is negative then $B_{i}=B^{\prime}$. If this value is positive then a new blocking term must be computed. We must determine if the blocking term will be from the inner segment or the final segment of task $\tau_{\mathrm{m}}$. If $\mathrm{U}-\mathrm{W}>\mathrm{V}$, then set $\mathrm{B}_{\mathrm{i}}=\mathrm{U}$ and remove task $\tau_{m}$ from $S P_{i 1}$; otherwise set $B_{i}=V$ and $\tau_{m}$ remains in $\mathrm{SP}_{\mathrm{i} 1}$.

Step 2: Determine how many jobs of task $\tau_{\mathrm{i}}$ must be checked. 
1. The length of the task- $\tau_{\mathrm{i}}$ busy period is:

$$
\begin{gathered}
L_{i}=\min \left(t>0 \mid B_{i}+\sum_{\tau_{p} \in M P_{i 1}}\left\lceil t / T_{p}\right\rceil C_{p}+\right. \\
\left.\sum_{\tau_{p} \in S P_{i 1}} C_{p}^{h}+\left\lceil t / T_{i}\right\rceil C_{i}=t\right)
\end{gathered}
$$

The length of the $\tau_{\mathrm{i}}$-busy period is determined by considering processing contributed by:

- the blocking term,

- the multiply preemptive tasks (relative to the first subtask),

- the singly preemptive tasks (relative to the first subtask), and

- complete jobs of $\tau_{\mathrm{i}}$.

The idea is to look for the minimum time $t$, such that all work of priority $\mathrm{P}_{\mathrm{i}}$ or higher initiated in the interval $[0, t]$ is completed at time $t$. Notice that multiply preemptive tasks and $\tau_{\mathrm{i}}$ may contribute processing more than once during the $\tau_{i}$-busy period, and the blocking term and singly preemptive tasks contribute exactly once during this busy period.

2. The number of jobs of $\tau_{\mathrm{i}}$ in the busy period is:

$$
N_{i}=\left\lceil L_{i} / T_{i}\right\rceil
$$

Step 3: Check the completion time of each of the $\mathrm{N}_{\mathrm{i}}$ jobs in the $\tau_{\mathrm{i}}$-busy period.

1. The completion time of the first subtask of the $\mathbf{k}^{\text {th }}$ job of task $\tau_{\mathrm{i}}$ is represented by $\mathrm{E}_{\mathrm{i} 1}(\mathrm{k})$.

$$
\begin{gathered}
E_{i 1}(k)=\min \left(t>0 \mid B_{i}+\sum_{\tau_{p} \in M P_{i 1}}\left\lceil t / T_{p}\right\rceil C_{p}+\right. \\
\left.\sum_{\tau_{p} \in S P_{i 1}} C_{p}^{h}+(k-1) C_{i}+C_{i 1}=t\right)
\end{gathered}
$$

To understand this equation, consider as an example the first subtask of the first job in canonical form. Under worst-case phasing, the completion of this subtask is impacted by the blocking term, all higher priority processing that is initiated at the same instant and the execution time of the subtask itself. The right side of the equation represents the first point in time t, where all of the processing initiated by multiply preemptive and single preemptive tasks in the interval $[0, t]$, the processing associated with the blocking term, and the processing associated with the subtask itself has completed.

2. Given the completion time of the first subtask of the $\mathbf{k}^{\text {th }}$ job, it is possible to calculate the completion of the second subtask of the $\mathbf{k}^{\text {th }}$ job. The first step in this calculation is to insert the correct elements into the set $\mathrm{SP}_{\mathrm{i} 2}$. Insert into $\mathrm{SP}_{\mathrm{i} 2}$ those tasks that were multiply preemptive relative to subtask $\tau_{i 1}$, but due to the higher priority of $\tau_{i 2}$, are singly preemptive relative to $\tau_{\mathrm{i} 2}$.

$$
\begin{aligned}
S P_{i 2}= & \left\{\tau_{p} \mid \tau_{p} \in\left(M P_{i 1}-M P_{i 2}\right) \wedge\right. \\
& \left.\left(\exists l \mid\left(P_{p 1}, \ldots, P_{p l} \geq P_{i 2}\right) \wedge\left(P_{p l+1}<P_{i 2}\right)\right)\right\}
\end{aligned}
$$

$\mathrm{SP}_{\mathrm{i} 2}$ is constructed by first selecting those tasks from $\mathrm{MP}_{\mathrm{i} 1}$ that may have become singly preemptive. This is accomplished via the set subtraction in the equation. Secondly, one must ensure that each of the selected tasks is actually a singly preemptive task relative to $\tau_{\mathrm{i} 2}$. This is accomplished by making sure that there exists a leading segment that has a priority greater or equal to $\mathrm{P}_{\mathrm{i} 2}$. Notice that tasks in $\mathrm{SP}_{\mathrm{i} 1}$ are not included in $\mathrm{SP}_{\mathrm{i} 2}$. This is because these tasks only preempt once during a $\tau_{\mathrm{i}}$-busy period and this single preemption has already been accounted for in the calculation of $E_{i 1}$.

3. Using the completion time of the first subtask and sets $\mathrm{SP}_{\mathrm{i} 2}$ and $\mathrm{MP}_{\mathrm{i} 2}$, the completion time of the second segment is:

$$
\begin{aligned}
& E_{i 2}(k)=\min \left(t>0 \mid E_{i 1}(k)+\right. \\
& \sum_{\tau_{p} \in M P_{i 2}}\left[\left\lceil t / T_{p}\right\rceil-\left\lceil E_{i 1}(k) / T_{p}\right\rceil\right] C_{p}+ \\
& \left.\sum_{\tau_{p} \in S P_{i 2}} \min \left(1,\left[\left\lceil t / T_{p}\right\rceil-\left\lceil E_{i 1}(k) / T_{p}\right\rceil\right]\right) C_{p}^{h}+C_{i 2}=t\right)
\end{aligned}
$$

The equation uses the completion time of the first subtask (in canonical form) as the starting point for calculating the completion time of the second subtask. The first summation represents the multiply preemptive processing initiated by tasks after the completion of the first subtask. The second summation represents the singly preemptive processing initiated by tasks after the completion of the first subtask. The "min" function within the second summation ensures that singly preemptive tasks can have at most one preemption effect on the remainder of the job. The execution time of the second subtask is then added in.

4. In general, given the completion time of the $\mathrm{j}^{\text {th }}$ subtask of the $k^{\text {th }}$ job, it is possible to calculate the completion of the $(j+1)^{\text {st }}$ subtask of the $k^{\text {th }}$ job.

Once again we must first insert the proper elements into the set $\mathrm{SP}_{\mathrm{ij}+1}$.

$$
\begin{aligned}
& S P_{i j+1}^{\prime}=\left\{\tau_{p} \mid \tau_{p} \in S P_{i j} \wedge\right. \\
& \quad\left(\left\lceil E_{i j}(k) / T_{p}\right\rceil-\left\lceil E_{i j-1}(k) / T_{p}\right\rceil\right)=0 \wedge \\
& \left.\quad\left(\exists l \mid\left(P_{p 1}, \ldots, P_{p l} \geq P_{i j+1}\right) \wedge\left(P_{p l+1}<P_{i j+1}\right)\right)\right\}
\end{aligned}
$$

$\mathrm{SP}_{\mathrm{ij}+1}^{\prime}$ is the set of singly preemptive tasks relative to $P_{i j}$ that have not yet exhibited their singly preemptive effect and are also singly preemptive relative to $\mathrm{P}_{\mathrm{ij}+1}$, and

$$
\begin{aligned}
& S P_{i j+1}^{\prime \prime}=\left\{\tau_{p} \mid \tau_{p} \in\left(M P_{i j}-M P_{i j+1}\right) \wedge\right. \\
& \left.\quad\left(\exists l \mid\left(P_{p 1}, \ldots, P_{p l} \geq P_{i j+1}\right) \wedge\left(P_{p l+1}<P_{i j+1}\right)\right)\right\}
\end{aligned}
$$


$\mathrm{SP}_{\mathrm{ij+1}}^{\prime \prime}$ is the set of multiply preemptive tasks relative to $P_{i j}$ that are singly preemptive relative to $P_{i j+1}$. The set $\mathrm{SP}_{\mathrm{ij}+1}$ is the union of the above two sets.

$$
S P_{i j+1}=S P_{i j+1}^{\prime} \cup S P_{i j+1}^{\prime \prime}
$$

The calculation for $\mathrm{E}_{\mathrm{ij}+1}(\mathrm{k})$ is:

$$
\begin{aligned}
& E_{i j+1}(k)=\min \left(t>0 \mid E_{i j}(k)+\right. \\
& \sum_{\tau_{p} \in M P_{i j+1}}\left[\left\lceil t / T_{p}\right\rceil-\left\lceil E_{i j}(k) / T_{p}\right\rceil\right] C_{p}+ \\
& \left.\quad \sum_{\tau_{p} \in S P_{i j+1}} \min \left(1,\left[\left\lceil t / T_{p}\right\rceil-\left\lceil E_{i j}(k) / T_{p}\right\rceil\right]\right) C_{p}^{h}+C_{i j+1}=t\right)
\end{aligned}
$$

All jobs of task $\tau_{i}$ will meet their deadlines if the following condition is satisfied.

$$
\max \left((k-1) T_{i}+D_{i}-E_{i m(i)}(k)\right) \geq 0 \text { for } k \leq N_{i}
$$

\subsection{Deadlines for Other Subtasks}

The analysis of the other subtasks is very similar (and in some cases identical) to the analysis of the final subtask. The only difference is in the analysis of the first job. Assume that subtask $\tau_{\mathrm{ij}}$ is to be analyzed, where $j \neq m(i)$. Let $P_{i}(j)=\min \left(P_{i k}, 1 \leq k \leq j\right)$. Special analysis of the first job of $\tau_{\mathrm{ij}}$ is necessary only if $P_{i}(j)>P_{i}$.

If the special analysis is needed, then truncate task $\tau_{i}$ after subtask $\tau_{i j}$ and use the algorithm for converting a task to canonical form on this truncated task. Determine task groupings for the truncated task and apply the stepwise method from the previous section. The schedulability test for the first job of subtask $\tau_{\mathrm{ij}}$ is: $D_{i j}-E_{i j}(1) \geq 0$

\section{Applying the Method to an Example}

\subsection{Problem Description}

We will use an example developed from a real-time robotics application to illustrate the utility of the theory developed in this paper and how to perform a schedulability analysis using the method described in the previous section. This example is derived from a real robot system that measures the shape of pipes inside a nuclear reactor, by moving around them and using a distance sensor. The task set corresponding to this system has been simplified to reflect only the important activities relevant to our analysis, and the numbers used are not exact, although they approximate the real magnitudes. With no loss of generality, we will consider all tasks to be periodic, by using a worst-case arrival assumption for those tasks whose nature is essentially aperiodic, namely that those tasks arrive at their maximum expected rates.

The system, which has five tasks, is represented in Figure 2. A sequence of tasks that execute serially at varying priorities are considered a single task with multiple subtasks, for our analysis. For example, task $\tau_{1}$ is considered as a single task, but is in fact composed of two system tasks, an interrupt service routine (ISR) and Servo Control, where Servo Control executes only as a consequence of being signaled by the ISR. The five tasks are:

- Robot control. Task $\tau_{1}$ has to control the robot's servomotors and has two subtasks, that have two different deadlines. The corresponding activities are: reading the inputs from the servo sensors and performing the control action for moving the robot.

- Measurement subsystem. Tasks $\tau_{2}$ and $\tau_{3}$ constitute the measurement subsystem, and synchronize with each other: $\tau_{2}$ reads the distance sensors and does some data preprocessing, while $\tau_{3}$ does some more processing and sends the results to a remote system.

- System command. Task $\tau_{4}$ is in charge of receiving and interpreting commands arriving from the remote system, while $\tau_{5}$ has to process and execute these commands. Both tasks synchronize with each other, and $\tau_{5}$ also has to update some control variables that affect the operation of the rest of the tasks.

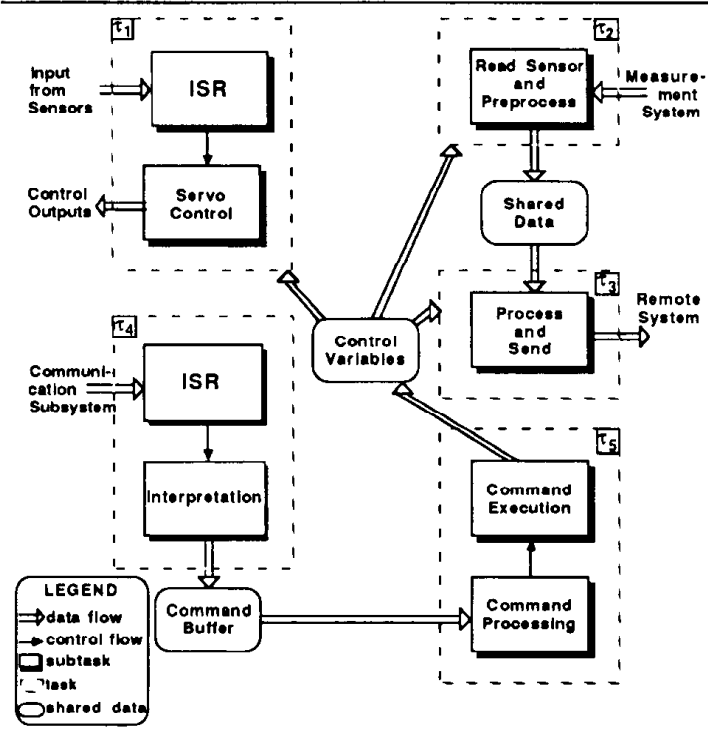

Figure 2: System Diagram

Hardware interrupts, synchronization, and existence of different deadlines lead to a task structure in which each task has several subtasks, each characterized by having different priorities and worst-case execution times. Priority ceiling protocol emulation [11] is being used for task synchronization and is responsible for the assignment of some of the priority levels. Table 1 shows the subtasks and characteristics of each task. All time values are in milliseconds.

- The lower level priorities of each task have been assigned according to rate monotonic order, using the 
task periods.

- Tasks $\tau_{1}$ and $\tau_{4}$ start with an ISR, and therefore have the priorities of their first sections fixed by the system's hardware.

- Tasks $\tau_{2}$ and $\tau_{3}$ synchronize with each other in their middle and final subtasks, respectively, and execute both of these subtasks at the same elevated priority. The reason for this elevated priority level will become apparent in the analysis phase.

- Tasks $\tau_{4}$ and $\tau_{5}$ also synchronize with each other, and are assumed to have their final and initial subtasks, respectively, executing at the same priority.

- Task $\tau_{5}$ 's final subtask must modify some control variables, and is therefore executed at high priority to prevent interference from some of the other tasks.

Table 1: Task Set Characteristics

\begin{tabular}{|c|c|c|c|c|c|c|c|c|c|}
\hline & \multicolumn{5}{|c|}{ Thing o foguirements } & \multicolumn{4}{|c|}{ Priorliy Structure } \\
\hline & $\mathbf{T}_{\mathbf{i}}$ & $C_{i}$ & $c_{12}$ & $C_{13}$ & $D_{i}$ & $P_{i_{1}}$ & $P_{\text {i2 }}$ & $P_{\text {is }}$ & Shape \\
\hline$\tau_{1}$ & 40 & 1 & 5 & - & 40 & 10 & 7 & - & \\
\hline$\tau_{2}$ & 100 & 10 & 5 & 5 & 100 & 4 & 8 & 4 & \\
\hline$\tau_{3}$ & 50 & 8 & 12 & - & 50 & 5 & 8 & - & \\
\hline$\tau_{4}$ & 200 & 10 & 20 & 3 & 200 & 9 & 2 & 3 & \\
\hline$\tau_{5}$ & 400 & 2 & 12 & 10 & 400 & 3 & 1 & 6 & $\neg$ \\
\hline
\end{tabular}

Although tasks $\tau_{1}$ and $\tau_{4}$ start with an ISR and could potentially have a self-preemption effect and violate Assumption 3, the fact that their final deadlines are before or at the end of their periods ensures that this effect cannot happen, if task deadlines are met. Therefore, all of the analysis developed in this paper applies to these two tasks, as long as they meet their deadlines.

Each task has its final deadline at the end of its period, and task $\tau_{1}$ has an additional deadline for its first subtask, due to physical constraints on the sensors: $D_{11}=1 \mathrm{~ms}$. The total CPU utilization is $97.5 \%$. Our goal is to derive a worst-case phasing or critical instant for each task, to be able to analyze the worst-case response times. In this way we can determine if the timing requirements of each task, and of the required associated subtasks, can be met under all circumstances.

\subsection{Problem Solution}

Before starting the analysis of each task we will reduce it to its canonical form. The first step in the analysis will be to determine the critical instant phasing, by identifying the blocking term and the multiply preemptive and singly preemptive sets. The second step is to obtain the number of jobs that have to be checked by evaluating the length of the task's busy period. Finally, the worst-case completion time for each job will be obtained by application of the schedulability equations, and checked against the deadlines or timing requirements.

3.2.1. Analysis of $\tau_{1}$ : The transformed canonical form of $\tau_{1}$ is obtained by lowering the priority of its first subtask, and combining the resultant equal priority subtasks into a single segment with priority 7 .

Step 1. The lowest priority level in task $\tau_{1}^{\prime}$ is 7 . We will classify the rest of the tasks according to this priority level to determine the critical instant phasing.

$\tau_{2}: \quad$ It is an LHL task (type 4), and its only contribution to the critical instant may be a blocking segment.

$\tau_{3}$ : It is an LH task (also type 4). It can only contribute with a blocking segment.

$\tau_{4}$ : It is an HL task (type 2), so it is classified as a singly preemptive task.

$\tau_{5}:$ It is an $L$ task (type 5) and has no effect on the critical instant.

Consequently, we have task $\tau_{4}$ in the set of single preemptive tasks $\left(\mathrm{SP}_{11}\right)$ with its first subtask $\tau_{41}$ acting as the $\mathrm{H}$ segment, and $B_{1}=C_{32}$ as the maximum of the blocking terms.

Step 2. The length of the busy period is:

$$
\begin{aligned}
L_{1}= & \min \left(t>0 \mid B_{1}+\sum_{\tau_{p} \in M P_{11}}\left\lceil t / T_{p}\right\rceil C_{p}+\right. \\
& \left.\sum_{\tau_{p} \in S P_{11}} C_{p}^{h}+\left\lceil t / T_{1}\right\rceil C_{1}=t\right) \\
L_{1}= & \min \left(t>0 \mid 0+C_{32}+C_{41}+\left\lceil t / T_{1}\right\rceil C_{1}=t\right) \\
L_{1}= & 0+12+10+1 \cdot 6=28
\end{aligned}
$$

Therefore, there is only one job, $N_{1}=\left\lceil 28 / T_{1}\right\rceil=1$, of $\tau_{1}$ in the busy period. As it can be seen, when the task has only one segment of constant priority and the deadline is before or at the end of the period, there can only be a single job in the busy period if it makes its deadline. This fact can be used as a shortcut for this step of the analysis.

Step 3. As the transformed canonical form has only one segment and there is only one job in the busy period, the completion time and the busy period expressions become identical. Consequently:

$$
E_{1}(1)=28 \leq 40=D_{1}
$$

Intermediate Deadline. Task $\tau_{1}$ meets its final deadline, but it also has a deadline for its first subtask, which has to be checked. According to the schedulability rules, $\tau_{1}$ 's critical instant is valid for all jobs of this first subtask, 
except for the first one. For this first job we have to create a different critical instant, according to its own priority level. In this case, the first subtask has the highest priority in the system; therefore, all the rest of the tasks can be classified as low priority (type 5), and the completion time of this first subtask will be:

$$
E_{11}(1)=C_{11}=1 \leq 1=D_{11}
$$

3.2.2 Analysis of $\tau_{2}$ : The transformed canonical form for $\tau_{2}$ is a task with a single segment $\tau_{21}^{\prime}$ of priority 4 .

Step 1. For task $\tau_{2}$ we will classify the rest of the tasks according to its lowest priority level, which is 4 .

$\tau_{1}, \tau_{3}$ : They are $H$ tasks (type 1 ) and have to be included among the multiply preemptive tasks.

$\tau_{4}$ : It is an HL task (type 2), so it is classified into the singly preemptive set.

$\tau_{5}:$ It is an LH task (type 4) and has a potential blocking effect on $\tau_{2}$.

Thus, we have tasks $\tau_{1}$ and $\tau_{3}$ in the set of multiply preemptive tasks $\left(\mathrm{MP}_{21}\right), \tau_{41}$ as a singly preemptive segment, and $B_{2}=C_{53}$ as the only blocking term.

Step 2. The length of the busy period is:

$$
\begin{aligned}
L_{2}= & \min \left(t>0 \mid B_{2}+\sum_{\tau_{p} \in M P_{21}}\left\lceil t / T_{p}\right\rceil C_{p}+\right. \\
& \left.\sum_{\tau_{p} \in S P_{21}} C_{p}^{h}+\left\lceil t / T_{2}\right\rceil C_{2}=t\right) \\
L_{2}= & \min \left(t>0 \mid C_{53}+\left\lceil t / T_{1}\right\rceil C_{1}+\left\lceil t / T_{3}\right\rceil C_{3}+\right. \\
& \left.C_{41}+\left\lceil t / T_{2}\right\rceil C_{2}=t\right) \\
L_{2}= & 10+3 \cdot 6+2 \cdot 20+10+1 \cdot 20=98
\end{aligned}
$$

Finding the minimum $t>0$ that satisfies the equation above can be accomplished by supposing an initial positive but very small value for $t$, obtaining the ceiling functions, and adding all the terms in the left-hand side of the equality. Using this result as the new value for $t$, we repeat the same process until we find a value of $t$ that is the same as in the last iteration and, therefore, satisfies the equality. If the total utilization is less than or equal to $100 \%$, then we know that the busy period will end (and so will the algorithm). In this particular case, we find that there is only one job of $\tau_{2}$ in the busy period.

Step 3. As the transformed canonical task has only one segment of constant priority, the completion time has the same expression as the length of the busy period. Therefore:

$$
E_{2}(1)=98 \leq 100=D_{2}
$$

Task $\tau_{2}$ also meets its final deadline.

3.2.3. Analysis of $\tau_{3}$ : Task $\tau_{3}$ is already in canonical form.

Step 1. Task $\tau_{3}$ has its basic priority at level 5 , and the classification of the rest of the tasks is:

$\tau_{1}$ : It is an $\mathrm{H}$ task (type 1) and is included among the multiply preemptive tasks.

$\tau_{2}$ : It is an LHL task (type 4), so its $\mathrm{H}$ segment has a potential blocking effect.

$\tau_{4}: \quad$ It is an HL task (type 2), so it is classified as a singly preemptive task.

$\tau_{5}$ : It is an LH task (type 4) and has a potential blocking effect on $\tau_{2}$.

Thus, we have task $\tau_{1}$ in the set of multiply preemptive tasks $\left(\mathrm{MP}_{31}\right), \tau_{41}$ as a singly preemptive segment, and $B_{3}=C_{53}$ as the maximum blocking term.

Step 2. The length of its busy period is:

$$
\begin{aligned}
L_{3}= & \min \left(t>0 \mid B_{3}+\sum_{\tau_{p} \in M P_{31}}\left\lceil t / T_{p}\right\rceil C_{p}+\right. \\
& \left.\sum_{\tau_{p} \in S P_{31}} C_{p}^{h}+\left\lceil t / T_{3}\right\rceil C_{3}=t\right) \\
L_{3}= & \min \left(t>0 \mid C_{53}+\left\lceil t / T_{1}\right\rceil C_{1}+C_{41}+\left\lceil t / T_{3}\right\rceil C_{3}=t\right) \\
L_{3}= & 10+2 \cdot 6+10+2 \cdot 20=72
\end{aligned}
$$

Consequently, there are two jobs, $N_{3}=\left\lceil 72 / T_{3}\right\rceil=2$, of $\tau_{3}$ in the busy period, which have to be checked.

Step 3. The completion time of the first segment of the first job is:

$$
\begin{aligned}
E_{31}(1)= & \min \left(t>0 \mid B_{3}+\sum_{\tau_{p} \in M P_{31}}\left\lceil t / T_{p}\right\rceil C_{p}+\right. \\
& \left.\sum_{\tau_{p} \in S P_{31}} C_{p}^{h}+C_{31}=t\right) \\
E_{31}(1)= & \min \left(t>0 \mid C_{53}+\left\lceil t / T_{1}\right\rceil C_{1}+C_{41}+C_{31}=t\right) \\
E_{31}(1)= & 10+1 \cdot 6+10+8=34
\end{aligned}
$$

For the second segment, we have to recalculate the sets of multiple and single preemptive tasks. $\mathrm{MP}_{32}$ will be empty, as there are no tasks with all their subtasks with priority higher than or equal to $8 . \mathrm{SP}_{32}$ can get elements from the tasks that were originally in $\mathbf{M P}_{31}$ and are not in $\mathrm{MP}_{32}$, if their priority is first higher and then lower than $\mathrm{P}_{32}$. Task $\tau_{1}$ satisfies these conditions and therefore the analysis for this subtask is:

$$
\begin{aligned}
& E_{32}(1)=\min \left(t>0 \mid E_{31}(1)+\right. \\
& \quad \sum_{\tau_{p} \in M P_{32}}\left[\left\lceil t / T_{p}\right\rceil-\left\lceil E_{31}(1) / T_{p}\right\rceil\right] C_{p}+ \\
& \left.\quad \sum_{\tau_{p} \in S P_{32}} \min \left(1,\left[\left\lceil t / T_{p}\right\rceil-\left\lceil E_{31}(1) / T_{p}\right\rceil\right]\right) C_{p}^{h}+C_{32}=t\right)
\end{aligned}
$$




$$
\begin{aligned}
E_{32}(1) & =\min \left(t>0 \mid E_{31}(1)+\right. \\
& \left.\min \left(1,\left[\left\lceil t / T_{1}\right\rceil-\left\lceil E_{31}(1) / T_{1}\right\rceil\right]\right) C_{11}+C_{32}=t\right) \\
E_{32}(1) & =34+1 \cdot 1+12=47 \leq 50=D_{3}
\end{aligned}
$$

And now we have to check the second job, in the same way:

$$
\begin{aligned}
E_{31}(2)= & \min \left(t>0 \mid C_{53}+\left\lceil t / T_{1}\right\rceil C_{1}+\right. \\
& \left.C_{41}+C_{3}+C_{31}=t\right) \\
E_{31}(2)= & 10+2 \cdot 6+10+20+8=60 \\
E_{32}(2)= & \min \left(t>0 \mid E_{31}(2)+\right. \\
& \left.\min \left(1,\left[\left\lceil t / T_{1}\right\rceil-\left\lceil E_{31}(2) / T_{1}\right\rceil\right]\right) C_{11}+C_{32}=t\right) \\
E_{32}(2)= & 60+0 \cdot 1+12=72 \leq 50+50=T_{3}+D_{3}
\end{aligned}
$$

We can see that task $\tau_{3}$ meets its deadlines through all of the busy period, thanks to the high priority of its last subtask. If the priority of this subtask had not been so high, let us say it remained at 5 , then the first job would have missed its deadline.

3.2.4. Analysis of $\tau_{4}$ : The transformed canonical form for $\tau_{4}$ is a task with two segments: $\tau_{41}^{\prime}$ of priority 2 and $\tau_{42}^{\prime}$ of priority 3 .

Step 1. Task $\tau_{4}$ has its lower priority subtask at level 2 , so we will classify the rest of the tasks accordingly.

$$
\begin{aligned}
& \tau_{1}, \tau_{2}, \tau_{3} \text { : } \\
& \text { They are } H \text { tasks (type 1) and their subtasks fall } \\
& \text { into the multiple preemptive set. } \\
& \tau_{5}: \quad \text { It is an HLH task (type 3), so its last segment has } \\
& \text { a potential blocking effect on } \tau_{4} \text {, and its first seg- } \\
& \text { ment a one-time preemptive effect. }
\end{aligned}
$$

Thus, we have tasks $\tau_{1}, \tau_{2}$, and $\tau_{3}$ in the set of multiply preemptive tasks $\left(\mathrm{MP}_{41}\right), \tau_{51}$ as a singly preemptive segment and $B_{4}=C_{53}$ as the only blocking term.

Step 2.The length of the busy period is:

$$
\begin{aligned}
L_{4}= & \min \left(t>0 \mid C_{53}+\left\lceil t / T_{1}\right\rceil C_{1}+\left\lceil t / T_{2}\right\rceil C_{2}+\right. \\
& \left.\left\lceil t / T_{3}\right\rceil C_{3}+C_{51}+\left\lceil t / T_{4}\right\rceil C_{4}=t\right) \\
L_{4}= & 10+5 \cdot 6+2 \cdot 20+4 \cdot 20+2+1 \cdot 33=195
\end{aligned}
$$

Consequently, there is only one job of $\tau_{4}$ in the busy period.

Step 3. We will now obtain the completion time of this job, starting with the first segment of its canonical form:

$$
\begin{aligned}
E_{41}^{\prime}(1)= & \min \left(t>0 \mid C_{53}+\left\lceil t / T_{1}\right\rceil C_{1}+\left\lceil t / T_{2}\right\rceil C_{2}+\right. \\
& \left.\left\lceil t / T_{3}\right\rceil C_{3}+C_{51}+C_{41}+C_{42}=t\right)
\end{aligned}
$$

$$
E_{41}^{\prime}(1)=10+5 \cdot 6+2 \cdot 20+4 \cdot 20+2+10+20=192
$$

For the completion time of the second segment of the canonical form, tasks $\tau_{1}, \tau_{2}$, and $\tau_{3}$ remain as multiple preemptive in the $\mathrm{MP}_{42}$ set. The analysis is:

$$
\begin{aligned}
& E_{42}^{\prime}(1)= \min \left(t>0 \mid E_{41}^{\prime}(1)+\right. \\
& {\left[\left\lceil t / T_{1}\right\rceil-\left\lceil E_{41}^{\prime}(1) / T_{1}\right\rceil\right] C_{1}+} \\
& {\left[\left\lceil t / T_{2}\right\rceil-\left\lceil E_{41}^{\prime}(1) / T_{2}\right\rceil\right] C_{2}+} \\
& {\left.\left[\left\lceil t / T_{3}\right\rceil-\left\lceil E_{41}^{\prime}(1) / T_{3}\right\rceil\right] C_{3}+C_{43}=t\right) } \\
& E_{42}^{\prime}(1)= 192+0+0+0+3=195 \leq 200=D_{4} \\
& \text { Task } \tau_{4} \text { also meets its final deadline. }
\end{aligned}
$$

3.2.5. Analysis of $\tau_{5}$ : The transformed canonical form for $\tau_{5}$ is a task with two segments: $\tau_{51}^{\prime}$ of priority 1 and $\tau_{52}^{\prime}$ of priorily 6.

Step 1. Task $\tau_{5}$ has its lower priority subtask at level 1, which is the lowest priority in the system, so all the rest of the tasks are of type 1 .

Step 2.The length of the busy period is:

$$
\begin{aligned}
L_{5}= & \min \left(t>0 \mid\left\lceil t / T_{1}\right\rceil C_{1}+\left\lceil t / T_{2}\right\rceil C_{2}+\left\lceil t / T_{3}\right\rceil C_{3}+\right. \\
& \left.\left\lceil t / T_{4}\right\rceil C_{4}+\left\lceil t / T_{5}\right\rceil C_{5}=t\right) \\
L_{5}= & 10 \cdot 6+4 \cdot 20+8 \cdot 20+2 \cdot 33+1 \cdot 24=390
\end{aligned}
$$

Consequently, there is only one job of $\tau_{4}$ in the busy period.

Step 3. We will now obtain the completion time of this job. The analysis of the first segment of the canonical form is:

$$
\begin{aligned}
E_{51}^{\prime}(1)= & \min \left(t>0 \mid\left\lceil t / T_{1}\right\rceil C_{1}+\left\lceil t / T_{2}\right\rceil C_{2}+\left\lceil t / T_{3}\right\rceil C_{3}+\right. \\
& \left.\left\lceil t / T_{4}\right\rceil C_{4}+C_{51}+C_{52}=t\right) \\
E_{51}^{\prime}(1)= & 5 \cdot 6+2 \cdot 20+4 \cdot 20+1 \cdot 33+2+12=197
\end{aligned}
$$

For the analysis of the second segment $\tau_{1}$ remains as a multiply preemptive task, $\tau_{41}$ is a singly preemptive segment, and the rest of the tasks have no influence. Therefore:

$$
\begin{aligned}
E_{52}^{\prime}(1)= & \min \left(t>0 \mid E_{51}^{\prime}(1)+\left[\left\lceil t / T_{1}\right\rceil-\left\lceil E_{51}^{\prime}(1) / T_{1}\right\rceil\right] C_{1}+\right. \\
& \left.\min \left(1,\left[\left\lceil t / T_{4}\right\rceil-\left\lceil E_{51}^{\prime}(4) / T_{4}\right\rceil\right]\right) C_{41}+C_{53}=t\right) \\
E_{52}^{\prime}(1)= & 197+1 \cdot 6+1 \cdot 10+10=223 \leq 400=D_{5}
\end{aligned}
$$

Task $\tau_{5}$ also meets its final deadline, so the total task set is schedulable. 


\section{Theoretical Analysis}

In this section, we present the theoretical underpinnings that support the rules for schedulability analysis that were presented and used in Sections 2 and 3.

\subsection{Busy Period Analysis}

The focus of this section is finding the longest response time for a particular subtask $\tau_{i j}$. Once this has been found, we check whether it is less than the subtask deadline $\mathrm{D}_{i j}$. If so, then the subtask timing requirements will be met under all task phasings. We define $P_{i}$ to be $\min \left(\mathrm{P}_{i j}, 1 \leq j \leq m(i)\right)$. If we select any particular phasing of the $n$ tasks and consider the resulting processor execution sequence that evolves over time, we can partition this execution sequence into intervals of time of two types: $\tau_{i}$-busy periods, as defined in Section 1, and instants of $\tau_{i}$-idleness or intervals of $\tau_{i}$-idleness, during which tasks of priority less than $P_{i}$ (or no task at all) are processed. The execution of $\tau_{i}$ takes place solely within the $\tau_{i}$-busy period segments. Consequently, when checking any timing requirements associated with $\tau_{i}$, attention can be restricted to $\tau_{i}$-busy periods.

The definition of a $\tau_{i}$-busy period permits two such periods to be "back-to-back." In this case, we consider the interval of lower priority processing or idleness to exist, but to be of zero length. One could require that the $\tau_{i}$-busy period have strictly positive intervals of lower priority processing (or idleness) on each side; however, this can lead to unnecessary deadline checking.

We begin our schedulability analysis by looking only at the final subtask deadlines, $\mathrm{D}_{i m(i)}, 1 \leq i \leq n$. We later discuss the deadlines for the earlier subtasks in the task set.

\subsection{Schedulability of the Canonical Form}

We next show that for purposes of checking its timing requirements, $\tau_{i}$ can be reduced to its canonical form. Consider any task $\tau_{i}$ that has two consecutive subtasks $\tau_{i j}$ and $\tau_{i j+1}$ with strictly decreasing priorities $\mathrm{P}_{i j}>\mathrm{P}_{i j+1}$. We consider a modified version of $\tau_{i}, \tau_{i}^{\prime}$, obtained by reducing the priority of $\tau_{i j}$ to $\mathrm{P}_{i j}^{\prime}=\mathrm{P}_{i j+1}$. We next show that the completion times of $\tau_{i}$ and of the subtasks $\tau_{i k}, j+1 \leq k \leq$ $m(i)$, are unchanged in the modified version.

Theorem 1: Suppose $\tau_{i}$ has two consecutive subtasks $\tau_{i j}$ and $\tau_{i j+1}$ of strictly decreasing priority $P_{i j}>P_{i j+1}$. Then for any task set phasing, the completion times of a task $\tau_{i}$ and its subtasks $\tau_{i k}, j+1 \leq k \leq m(i)$ are unchanged if the priority of $\tau_{i j}$ is reduced to $P_{i j+1}$, assuming all equal priority segments are executed in the same relative order.

Proof: Let the execution sequence for the task set be given. For any single job of $\tau_{i}\left(\tau_{i}^{\prime}\right)$ let $b_{i k}\left(b_{i k}^{\prime}\right)$ and $f_{i k}\left(f_{i k}^{\prime}\right)$ denote the start time and the completion time of $\tau_{i k}\left(\tau_{i k}^{\prime}\right)$. Notice that the completion time of $\tau_{i k}\left(\tau_{i k}^{\prime}\right)$ is the activation time $o \tau_{i k+1}\left(\tau_{i k+1}^{\prime}\right)$. The activation time is the instant at which a particular task or subtask becomes ready to execute. Preemption may cause the task to start executing at an instant later than the activation; we call this the start time. Assume that the two versions of this job of $\tau_{i}$ are activated at the same time. The execution sequences will then be identical up to $f_{i j-1}$. If we prove $b_{i j+1}=b_{i j+1}^{\prime}$, then the execution sequences will be identical after $b_{i j+1}$, and the result will follow, assuming that segments with equal priority are processed in the same order in the original and in the modified task set. For the original task set, the interval $\left[f_{i j-1}, b_{i j+1}\right]$ consists of the execution of $\tau_{i j}$ and other tasks of higher or equal priority than $\tau_{i j+1}$. At time $b_{i j+1}$, all work of equal or higher priority than $\tau_{i j+1}$ will have just finished, and that subtask can begin. If we now reduce the priority of $\tau_{i j}$ to that of $\tau_{i j+1}$, then exactly the same work will be done during this interval, although possibly in another order. At time $b_{i j+1}$, all work of equal or higher priority than $\tau_{i j+1}$ will also have just finished, and that subtask will begin execution. Consequently, $\mathrm{b}_{i j+1}$ $=b_{i j+1}^{\prime}$, and the results hold. Moreover, since the activation time of both versions is the same at the beginning of any $\tau_{i}$-busy period, this result holds for the entire busy period.

The above theorem can be used to simplify the determination of whether a particular task meets its final deadline. If any consecutive subtasks are of strictly decreasing priority, we can reduce the priority of the first to that of the second and merge these two into a single segment. By applying this theorem to all such consecutive subtasks, we can reduce the task to canonical form, as defined in Section 2 .

\subsection{Worst-Case Phasing}

Recall that $\mathrm{P}_{i}=\min \left(\mathrm{P}_{i j}, 1 \leq j \leq m(i)\right)$ and that the execution sequence arising from any choice of task phasings will create an alternating sequence of $\tau_{i}$-busy periods and periods of less than priority level $\mathrm{P}_{i}$ execution. We select a $\tau_{i}$-busy period and seek to determine the task phasings that will create the longest response time for subtask $\tau_{i m(i)}$.

Theorem 2: The longest response time for $\tau_{i m(i)}$ is found during a $\tau_{i}$-busy period initiated by $\tau_{i}$.

Proof: Suppose that $\tau_{i}$ does not initiate a $\tau_{i}$-busy period, so there exists an interval of execution of length $A$ $>0$ prior to the first initiation of $\tau_{i}$ during which subtasks of priority level $\mathrm{P}_{i}$ or higher are continuously executed. If the initiation time of $\tau_{i}$ were noved back by $A$, then $\tau_{i m(i)}$ could not start its execution any earlier than it did with its original initiation time, because all subtasks that were initiated during the period of length $A$ would still need to be executed before $\tau_{i m(i)}$ can begin execution. Thus, the response times for all jobs of $\tau_{i m(i)}$ would be increased by $A$. Thus, the situation in which $\tau_{i}$ does not initiate its $\tau_{i}$-busy period may not give the worst-case response time.

According to Theorem 1, one can reduce $\tau_{i}$ to its transformed canonical form. The resulting task, $\tau_{i}^{\prime}$, will consist 
of $m(i)$ subtasks $\tau_{i 1}^{\prime}, \ldots, \tau_{i m(i)}^{\prime}$ having priorities $\mathrm{P}_{i}=\mathrm{P}_{i 1}^{\prime}<\ldots<\mathrm{P}_{i m(i)}^{\prime}$. The transformed canonical form is useful for reasoning about the phasing of the rest of the tasks that creates the worst-case response. This phasing will depend on the priority levels of each task, compared to the priority of the first segment of the canonical form task, resulting in the classification of tasks by type that appeared in Section 2.

Clearly, type 5 tasks (lower priority) cannot be executed during a $\tau_{i}$-busy period, and therefore cannot influence the response time of $\tau_{i}$. We now seek the phasing of the 4 remaining task types that will maximize the longest response time for $\tau_{i}$ during the busy period.

4.3.1. Type 1 Tasks $(\mathrm{H})$ : The phasing of a type 1 task that will create the largest response time for any job of $\tau_{i}$ in a $\tau_{i}$-busy period is to have such a task initiated at the same instant that $\tau_{i}$ is initiated. To see this, suppose that the first job of such a task within the $\tau_{i}$-busy period is initiated at time $I>0$. The cumulative processor demands from this task during $[0, t]$ for every $t \in[0, b)$ monotonically increase as $I$ decreases towards 0 . Consequently, these demands are maximized uniformly over time by choosing $\mathrm{I}=0$; this maximizes the completion time of all subtasks and all jobs of $\tau_{i}$ in the busy period. Therefore, setting $I=0$ for any type 1 task will lead to the longest response time of $\tau_{i}$.

4.3.2. Type 4 Tasks $\left((\mathrm{LH})^{+} \mathrm{L}^{0}\right)$ : All $\mathrm{H}$ segments in type 4 tasks are preceded by an L segment that has priority lower than $P_{i}$. This means that, as tasks cannot suspend themselves, at most one of the $\mathrm{H}$ segments can be processed during the $\tau_{i}$-busy period. Furthermore, only one segment of one of the type 4 tasks can be processed during this entire busy period. For $\tau_{i}$ to initiate its own busy period, and for a segment of a type 4 task to execute during the busy period, the type 4 task must be executing one of its high-priority segments when $\tau_{i}$ is initiated. It can finish that segment, but no further processing is possible until the busy period ends. Thus, to determine the worst-case phasing for all type 4 tasks, we must consider all of the $\mathrm{H}$ segments of all of these tasks and select the one with the longest processing requirement. That segment should be starting just as $\tau_{i}$ is initiated. The phasing of the other tasks is irrelevant. We let $B^{\prime}$ denote the length of this longest segment.

4.3.3. Type $2\left((\mathrm{HL})^{+}\right)$and Type $3\left((\mathrm{HL})^{+} \mathrm{H}\right)$ Tasks: Type 2 tasks are similar to type 4 , in that only one segment of the task can be processed during the busy period. The difference is that by phasing each type 2 task to be initiated at time 0 , each will contribute a processing requirement that must be finished during the busy period, in contrast to the type 4 tasks, which collectively contribute only a single processing requirement. However, one of the type 2 tasks may have a long blocking segment, so long that it contributes more processing requirements than its initial segment would contribute; we have to determine if this is the case.

Type 3 tasks are the most complicated to phase. Only part of a type 3 task can be processed during the $\tau_{i}$-busy period, but that part can be an inner $\mathrm{H}$ segment, the last $\mathrm{H}$ segment, the first $\mathrm{H}$ segment, or the last followed by the first $\mathrm{H}$ segment. It should be noticed, however, that the inner $\mathrm{H}$ and final $\mathrm{H}$ segments are blocking terms and, as tasks do not suspend themselves, at most one blocking term chosen from the type 2, 3, and 4 tasks can be executed during the busy period.

To determine which task, if any, of types 2 and 3 acts with a blocking segment, and therefore has to be phased with $\tau_{i}$ at one of its inner or final $\mathrm{H}$ segments, we can follow the next procedure. For each type 2 and type 3 task, let $W$ be the computational requirement of the initial $\mathrm{H}$ segment, $U$ be the requirement of the longest inner $\mathrm{H}$ segment (if any) and $V$ be the computation requirement of the final $\mathrm{H}$ segment (for type 3 tasks). If there is no inner blocking segment then set $U=0$, and if there is no final blocking segment then set $V=0$.

Assume that each type 2 or type 3 task is initiated at the same time as task $\tau_{i}$. Now consider the two other possible phasings:

1. Initiating the longest inner $\mathrm{H}$ segment at the same time as $\tau_{i}$ will increase $\tau_{i}$ 's response times by $U-W-B^{\prime}$, which is the marginal gain in total computational requirement from using this inner $\mathrm{H}$ segment.

2. For type 3 tasks, initiating the final $\mathrm{H}$ segment at the same time as $\tau_{i}$ may increase $\tau_{i}$ 's response times by $V-B^{\prime}$. For type 2 tasks this value is negative or zero (because $V=0$ ), and will be disregarded in the next steps. Note that this is a pessimistic estimate in that it assumes that the final segment serves as a blocking segment and the initial segment is a preempting segment. However, the busy period may end before the initial segment can preempt.

For each type 2 and type 3 task, we calculate the gain in latency as $\max \left[\left(U-W-B^{\prime}\right),\left(V-B^{\prime}\right)\right]$, and we choose the task with the largest value. If this value is negative, then all of these tasks should be initiated at the same time as $\tau_{i}$, and the blocking term would be $\mathrm{B}_{i}=B^{\prime}$. If the largest value is positive, then either the inner blocking or final blocking segment should be selected. If $U-W \geq V$, then the inner segment is chosen, and $\mathrm{B}_{i}=U$; in this case the initial $\mathrm{H}$ segment does not contribute a preemption effect. If $U-W$ $<V$ then the final segment is chosen, we set $\mathrm{B}_{i}=V$, and this task also remains as a singly preemptive task. All the other tasks should be initiated at the same time as $\tau_{i}$.

\subsection{Other Subtask Deadlines}

The preceding analysis was carried out under the assumption that each task had only a single deadline at the end of its final subtask. We now wish to allow additional 
subtask deadlines, for example $\tau_{i j}$ having a deadline $D_{i j}$. Clearly, one must check the deadlines of each of these subtasks throughout the $\tau_{i}$-busy period for the worst-case phasing developed earlier. However, this is not sufficient, because it will only ensure that jobs of subtasks after the first job in the busy period will meet their worst-case timing requirement. It does not guarantee the first job, because the phasing may not be worst for the first job of the subtask. The reason for this is that the phasing developed was based on the $\tau_{i}$-busy period in which only activity of priority $\mathrm{P}_{i}$ or higher is executed, $\mathrm{P}_{i}$ being the minimum priority of all of the subtasks of $\tau_{i}$. However, the appropriate priority level against which to create a longest response time phasing for the first job should be based upon a possibly higher level priority, $\mathrm{P}_{i}(\hat{N})=\min \left(\mathrm{P}_{i k}, 1 \leq k\right.$ $\leq j$ ). This is the same as thinking of a $\tau_{i}^{j}$-busy period, where task $\tau_{i}^{j}$ is a task composed of the first $j$ subtasks of $\tau_{i}$.

Theorem 3: The deadline, $D_{i j}$, of $\tau_{i j}$ will be met under all task phasings provided:

1. The deadline of the first job of $\tau_{i j}$ is met under the worst-case phasing for a $\tau_{i}^{j}$-busy period, and

2. The deadline of all jobs of $\tau_{i j}$ after the first are met during a $\tau_{i}$-busy period with worst-case phasing for the minimum priority level of that busy period.

Proof: For cach subtask processed during the $\tau_{\text {-busy }}$ period, we record the minimum priority of it and all subtasks of $\tau_{i}$ processed before it during this busy period. This minimum priority is defined as $\mathrm{P}_{i}(j)$ for the first job of each subtask of $\tau_{i}$ and will be $P_{i}$ for all subtasks of second and later jobs. The proof has two parts: finding the longest response time for jobs with minimum priority $P_{i}$ and finding the longest response time for jobs with minimum priority $\mathrm{P}_{i}(j)>\mathrm{P}_{i}$.

The case of deadlines for subtasks with minimum priority $\mathrm{P}_{i}$ follows easily from the reasoning given in Section 4.3. Specifically, type 5 tasks can be ignored, type 1 tasks should be phased to maximize preemption as should any type 2 and type 3 tasks chosen for preemption. The largest blocking term derived from type 2,3 , and 4 tasks will also be the same, because a subtask of priority level $\mathrm{P}_{i}$ prior to the subtask in question is being blocked to the maximum extent, and this maximum blocking time will also delay all subsequent subtasks of $\tau_{i}$. Consequently, one needs only to check the deadlines of all subtasks during the $\tau_{i}$-busy period using the worst-case phasing derived in Section 4.3.

Subtasks $\tau_{i j}$ of the first job of $\tau_{i}$ with $P_{i}(j)$ greater than $P_{i}$ must be handled separately, because their critical instant phasing can be different. One must now derive the worst-case phasing described in Section 4.3 for type $1-4$ tasks, except the priority level $P_{i}$ must be replaced by $P_{i}(j)$. Consider first the subtask $\tau_{i k}$ with the largest $k$ among those for which $\mathrm{P}_{i}(k)>\mathrm{P}_{i}$. We wish to determine the longest response time for its first job. To do this, we ignore any later subtasks by considering only the task $\tau_{i}^{k}$, and reduce this truncated task to its canonical form. Note that the minimum priority is now $\mathrm{P}_{\mathrm{f}}(k)$ and the other $n-1$ tasks must be reclassified into the five types with respect to $\mathrm{P}_{i}(k)$. Once this is done, the arguments of Section 4.3 can be used to derive the worst-case phasing, and the longest response time of $\tau_{i k}$ can be computed. One now picks the subtask $\tau_{i k^{\prime}}, k^{\prime}<k$, with the next largest $k^{\prime}$ among those with $\mathrm{P}_{i}\left(k^{\prime}\right)>\min \left(P_{1}, \ldots, P_{k}\right)$, truncates $\tau_{i}$ there to obtain $\tau_{i}^{k}$, reduces it to canonical form, classifies the other tasks, and invokes the worst-case phasing to derive its worst-case response time. This process continues until all deadlines of first jobs have been checked. Thus conditions (1) and (2) are sufficient for all subtask deadlines to be met.

\subsection{Scheduling Bounds}

The example presented in Figure 1 was designed to show the added scheduling complexity that can occur when tasks are composed of subtasks that are executed at different fixed priority levels. This example also shows a scheduling benefit that can accrue from such a task structure. Using ordinary rate monotonic scheduling, a task set with two tasks: $C_{1}=4, T_{1}=10$ and $C_{2}=6, T_{2}=14$, is schedulable, but fully utilizes the processor with total utilization of .829 , essentially equal to the Liu and Layland bound of .828 for two tasks. However, the example in Section 1.2 modifies task two to have two subtasks of differing priority. This modified task set has a total utilization of .971 and is schedulable using a fixed priority algorithm. Thus, we see that we can increase the fixed priority schedulability of a task set by decomposing one or more of the tasks into subtasks that are executed at modified priority levels, and this can offer an increase in schedulability over that provided by the simple rate monotonic algorithm.

This paper is restricted to methods by which the schedulability of complex tasks can be determined for any fixed priority scheduling algorithm. The question of finding least upper bounds on processor utilization which generalize the Liu and Layland bounds will be addressed in a second paper. However, one simple and very interesting result for two tasks is that by dividing the one having the largest period into two subtasks, one with the lowest priority and one with globally highest priority, $100 \%$ schedulability can be attained. Specifically, given any two periodic tasks with $\mathrm{T}_{1}<\mathrm{T}_{2}$ and utilizations $\mathrm{U}_{1}+\mathrm{U}_{2}$ $=1$, one should decompose $\tau_{2}$ into two subtasks with $C_{21}$ $=\mathrm{C}_{2}-\left(\mathrm{T}_{1}-\mathrm{C}_{1}\right), \mathrm{C}_{22}=\mathrm{T}_{1}-\mathrm{C}_{1}$; these values are always non-negative. $\tau_{1}$ has intermediate priority, $\tau_{21}$ has lowest priority and $\tau_{22}$ has highest priority. The resulting task set is schedulable, the C's and T's are arbitrary and the task set has $100 \%$ utilization. For example, the task set 
depicted in Figure 1 with $C_{2}$ increased to 8.4 has $100 \%$ utilization, yet it is schedulable if the second task is broken into two subtasks with $\mathrm{C}_{21}=2.4, \mathrm{C}_{22}=6$, and $\tau_{22}$ is given highest priority. Ordinary rate monotonic scheduling can meet the timing requirements only if $\mathrm{C}_{2} \leq 6$.

\section{Conclusions}

Even when application-level tasks are assigned fixed priorities, the actual priority structure of a realistic system can be much more complex. Characteristics of the operating system and underlying hardware impact the priority structure and consequently the timing behavior of the system. For example, the task dispatching mechanism of the operating system, the interrupt architecture of the processor, synchronization protocols, and intertask communication mechanisms all contribute to the system's actual timing behavior. In order to accurately predict system behavior, these effects should be included in the schedulability equations that model the system's behavior.

This paper offers a generalized model of fixed priority scheduling that provides a theoretical framework for analyzing task sets scheduled through a fixed priority preemptive scheduler, where each task is comprised of a number of subtasks, each executing at a different priority level. For a set of tasks with a complex priority structure, we offer a method of analysis with an underlying theoretical foundation. For simple task sets, our contribution is a formalization of techniques currently being used [3]. Furthermore, the method shows that an increase in schedulability can be achieved by taking advantage of the high-priority execution of the final subtasks of a task.

Another very important highlight of this method is that it provides some simple techniques for reasoning about time in systems with varying priorities. The fact that tasks can be reduced to a canonical form simplifies analysis and allows one to easily reason about worst-case phasing. It also simplifies analysis by allowing the classification of tasks according to the priority of their first subtask. We feel that in practical problems the algorithm described in Section 2 is easily implemented and runs efficiently; however, the worst case complexity of this algorithm is an open question.

\section{References}

1. Borger, M. W., Klein, M. H., and Veltre, R. A. "RealTime Software Engineering in Ada: Observations and Guidelines". Software Engineering Institute Technical Review (1988).

2. Goodenough, J. B., and Sha, L. "The Priority Ceiling Protocol: A Method for Minimizing the Blocking of High Priority Ada Tasks". Proceedings of the 2nd International Workshop on Real-Time Ada Issues (June 1988).
3. Klein, M. H., and Ralya, T. An Analysis of Input/Output Paradigms for Real-Time Systems. Tech. Rept. CMU/SEI-90-TR-19, Software Engineering Institute, July 1990.

4. Lchoczky, J. P., and Sha, L. "Performance of RealTime Bus Scheduling Algorithms". ACM Performance Evaluation Review, Special Issue 14, 1 (May, 1986).

5. Lehoczky, J.P. "Fixed Priority Scheduling of Periodic Task Sets with Arbitrary Deadline". IEEE Real-Time System Symposium (1990).

6. Leung, J. and Whitehead, J. "On Complexity of Fixed-Priority Scheduling of Periodic Real-Time Tasks". Performance Evaluation 2, 237-50 (1982).

7. Liu, C.L., and Layland, J.W. "Scheduling Algorithms for Multi-Programming in a Hard Real-Time

Environment". Journal of the Association for Computing Machinery Vol. 20, 1 (January 1973), pp. 46-61.

8. Rajkumar, R., Sha, L., and Lehoczky, J.P. "Real-Time Synchronization Protocols for Multiprocessors". IEEE Real-Time Systems Symposium (December 1988).

9. Rajkumar, R. "Real-Time Synchronization Protocols for Shared Memory Multi-Processors". Proceedings of The 10th International Conference on Distributed Computing (1990).

10. Sha, L., Rajkumar, R., Lehoczky, J. and Ramamritham K. "Mode Change Protocols for Priority-Driven Preemptive Scheduling". The Journal of Real-Time Systems Vol. 1 (1989), pp. 243-264.

11. Sha, L. and Goodenough, J. B. "Real-Time Scheduling Theory and Ada". IEEE Computer Vol. 23, No. 4 (April 1990).

12. Sha, L., Rajkumar, R., and Lehoczky, J. P. "RealTime Scheduling Support in Futurebust". IEEE RealTime Systems Symposium (1990).

13. Sha, L., Rajkumar, R., and Lehoczky, J. P. "Priority Inheritance Protocols: An Approach to Real-time Synchronization". IEEE Transactions on Computers (Sept. 1990).

14. Sha, L., Klein, M. H., and Goodenough, J. B. Rate Monotonic Analysis for Real-Time Systems. In Foundations of Real-Time Computing: Scheduling and Resource Management, van Tilborg, Andre and Koob, Gary M., Ed., Kluwer Academic Publishers, 1991, pp. 129-155.

15. Sprunt, B., Sha, L., and Lehoczky, J.P. "Aperiodic Task Scheduling for Hard Real-Time Systems". The Journal of Real-Time Systems , 1 (1989), pp. 27-60. 